\title{
Synthesis and Biological Evaluation of FTY720 (Fingolimod) Derivatives with Aromatic Head Group as Anticancer Agents
}

\author{
Taeho Lee, ${ }^{a, \#}$ Hong Seop Moon, ${ }^{b, \#}$ Seon Woong Kim,${ }^{b}$ Jitendra Shrestha,${ }^{b}$ Sang Mi Shin, ${ }^{c}$ \\ Joo-Youn Lee, ${ }^{d, e}$ Sanghee Kim, ${ }^{d}$ Eun-Young Park, ${ }^{*}, b$ and Dong Jae Baek ${ }^{*, b}$ \\ ${ }^{a}$ College of Pharmacy, Research Institute of Pharmaceutical Sciences, Kyungpook National University; Daegu 41566, \\ South Korea: ${ }^{b}$ College of Pharmacy and Natural Medicine Research Institute, Mokpo National University; Jeonnam \\ 58554, South Korea: ${ }^{c}$ College of Pharmacy, Chosun University; Gwangju 61452, South Korea: ' College of Pharmacy, \\ Seoul National University; Seoul 08826, South Korea: and ${ }^{e}$ Korea Chemical Bank, Korea Research Institute of \\ Chemical Technology; Daejeon 34114, South Korea. \\ Received January 29, 2018; accepted July 5, 2018
}

FTY720 is employed for the treatment of multiple sclerosis and exerts apoptotic effects on various cancers through protein phosphatase $2 \mathrm{~A}$ (PP2A) activation. In compound 4, the dihydroxy head group of FTY720 was modified into dihydroxy phenyl group. The cell survival in compound 4 treated colorectal and gastric cancer cells was significantly reduced as compared with control, 34.6 and $25.1 \%$, respectively. The docking study of compound 4 showed that the aromatic head group effectively binds to PP2A.

Key words FTY720; fingolimod; anticancer; sphingosine kinase; derivative

FTY720 (also known as fingolimod, 1) is a synthetic compound, which is modified from myriocin (ISP-1), and it is an immunosuppressant that is effective against multiple sclerosis; its use has been approved by the Food and Drug Administration (FDA). FTY720 is structurally similar to natural lipid, sphingosine, and is phosphorylated in vivo to form FTY720phosphate. This drug has an inhibitory action on various cancers such as breast cancer and leukemia. ${ }^{1-3)}$ These anticancer activities are related to the activity of protein phosphatase $2 \mathrm{~A}$ (PP2A) regardless of phosphorylation. ${ }^{4)}$ In addition, FTY720 stimulates beta cell regeneration in mice to normalize hyperglycemia and produce insulin. ${ }^{5,6}$ Based on various medicinal effects of FTY720, the research has been ongoing until date. Continuous efforts are being put forward by researchers to design FTY720 derivatives (selective for certain diseases) with improved biological effects of FTY720.

FTY720 consists of a structurally polar head group, an aromatic backbone, and an aliphatic chain with 8 carbons. (Fig. 1) The derivatives known so far have been synthesized by modifying each of these three parts of FTY720. Recently, derivatives of FTY720 with modified aromatic part have been reported to be effective in the treatment of multiple sclerosis. ${ }^{7)}$ However, most of the derivatives retain the structure of the aromatic backbone. FTY720-OMe (ROME) is a derivative of FTY720 with selective introduction of one methoxy group into the head group (Fig. 1). ROME selectively inhibits sphingosine kinase (SK)2 and suppresses breast cancer cells. ${ }^{8)}$ Various compounds in the form of FTY720 inhibit SK. SK has two isoforms, SK1 and SK2, which are present in different compartments in the cell. SK inhibitors inhibit the production of sphingosine-1-phosphate (S1P) and thus exhibit anticancer activity. ${ }^{8)}$ SK1 and SK2 play different roles in vivo, therefore, selective inhibitors are required for drug development. ${ }^{9)}$ However, the enantioselective introduction of functional groups into the head of FTY720, such as ROME or FTY720

\footnotetext{
\# These authors contributed equally to this work.
}

$(S)$-phosphate, requires a complicated pathway and many synthetic steps. We have recently reported FTY720 derivatives and their chemical structure to contain serine structure similar to the sphingolipid head group. ${ }^{10)}$ In contrast, like SH-RF-177 or RB-042, synthesis of the derivatives by introducing the chiral head group, such as commercially available azacyclic structure or pyrrolidinol, is easy and biologically effective. SH-RF-177 exhibits anti-leukemic activity both in vitro and in vivo without affecting the $\mathrm{S} 1 \mathrm{P}$ receptor, and $\mathrm{RB}-042$ effectively inhibits sphingosine kinase 1 and $2 .^{11-13)}$ ST-968 is an oxazolo-oxazole derivative (butterfly analog) of FTY720 (Fig. 1) with three-dimensionally fixed head group and effectively binds to S1P1 as compared to FTY720. ${ }^{14)}$ Substitution of functional group in the aromatic structure is useful for finding the binding position of the target because they are fixed compared to the substitution in aliphatic or cyclic forms, which is flexible. Derivatives with the aromatic group in head group, such as compound $\mathbf{3 g}$ or RB-050 (Fig. 1), have not been developed much. Compound $\mathbf{3 g}$ has an antituberculosis effect, and RB-050 weakly inhibits SK1 and 2. ${ }^{13,15)}$ These derivatives are very easy to synthesize thus making it easy to figure out the

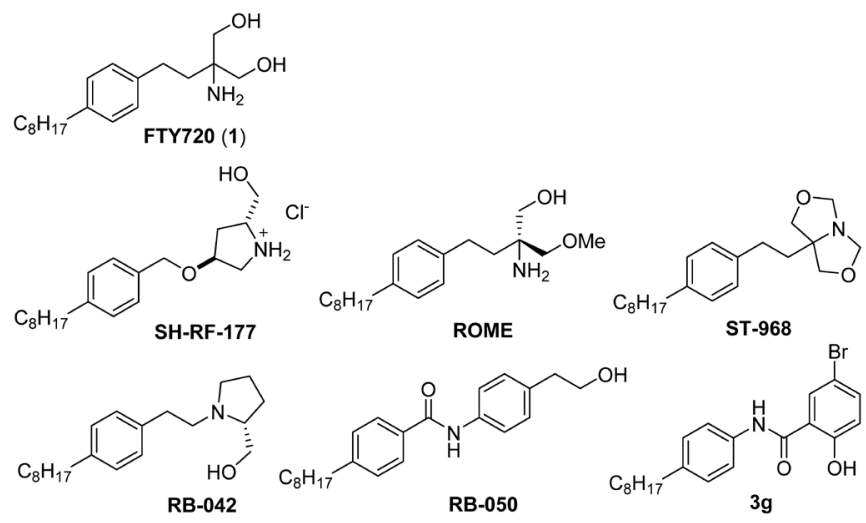

Fig. 1. FTY720 and Known Derivatives

* To whom correspondence should be addressed. e-mail: parkey@mokpo.ac.kr; dbaek@mokpo.ac.kr 
a)

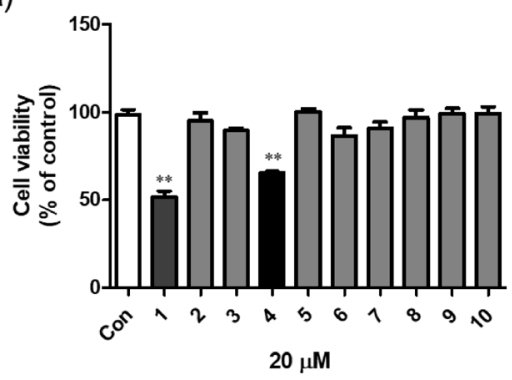

b)

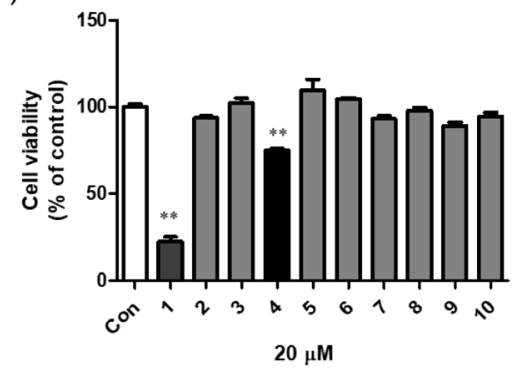

c)

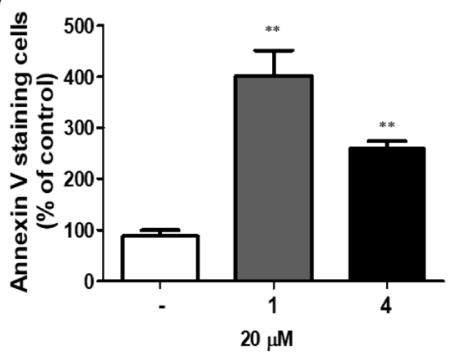

Fig. 2. Cytotoxic Effects of FTY720 (1) and Derivatives in Cancer Cells

Cell viability was measured in HT29 (a) and AGS (b) cells treated with $20 \mu \mathrm{M}$ FTY720 derivatives. (c) Apoptotic cells were stained with annexin-V-FITC. ** $p<0.01$ compared with control cells.
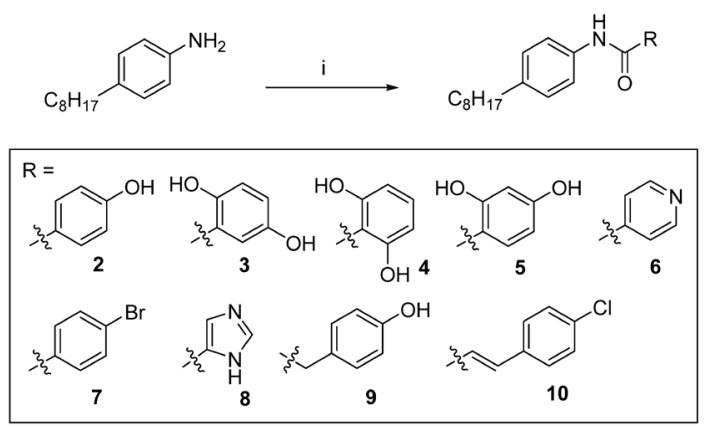

Reagents and condition: i) RCOOH, EDCI, DMAP, $\mathrm{CH}_{2} \mathrm{Cl}_{2}, 12 \mathrm{~h}$, r.t.

Chart 1. Synthesis of FTY720 Derivatives 2-10

structure-activity relationship of various functional groups substituted in the aromatic group.

For this reason, we have investigated the anticancer activity of FTY720 derivatives containing the aromatic head groups with various functional groups. Derivatives were synthesized using 1-ethyl-(3-3-dimethylaminopropyl)carbodiimide hydrochloride (EDCI) along with 4- $n$-octylaniline as starting material and compounds $\mathbf{2 - 1 0}$ was obtained by introducing the aromatic head group, such as benzoic acid and nicotinic acid (Chart 1). To investigate the anti-tumor activity of the synthesized FTY720 analogs, the degree of cell death was examined in HT29 cells (human colorectal adenocarcinoma cell) and AGS cells (human gastric adenocarcinoma cell). Statistically significant cell death was observed only in compound 4 (about 34.6 and 25.1\%) compared to the control, and no statistically significant cell death was observed with other analogs (Figs. 2a, b). We used annexin-V staining to determine whether the cell death induced by the compound 4 is the result of increased apoptosis. The number of apoptotic cells was significantly increased in HT29 cells treated with compound 4 for $24 \mathrm{~h}$ (Fig. 2c). Among the compounds 2-5 in which dihydroxyphenyl was introduced, only the compound 4 was found to be active, therefore it is apparent that the position of hydroxyl group seems to play an important role in exerting anticancer activity. In the case of ROME, an FTY720 derivative, there are immense activity changes even the small structural differences in the FTY 720 head group. Therefore, the selective anticancer activity of compound 4 provides valuable information in designing of novel FTY720 derivatives. Since FTY720 is known as a PP2A activator, we examined the PP2A activity of compound 4 in HT29 cells.

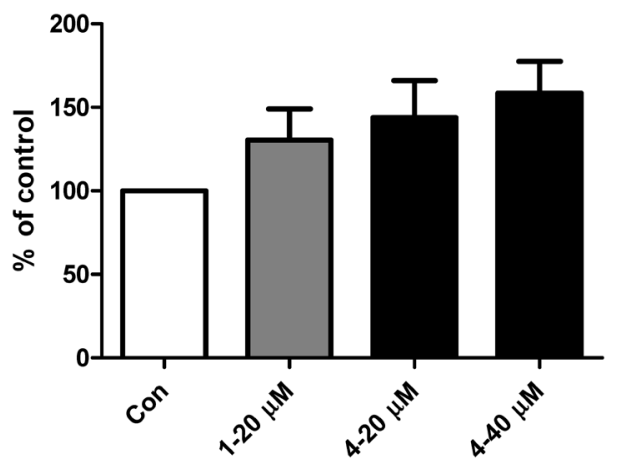

Fig. 3. PP2A Activity of FTY720 (1, Gray) and Compound 4 (Black) in HT29 Cells

HT29 cells were treated with FTY720 and compound $\mathbf{4}$ for $8 \mathrm{~h}$ and PP2A activity was measured.

FTY720 and compound 4 treatment increased PP2A activity at $8 \mathrm{~h}$ after treatment in HT29 cells. These results suggest that compound $\mathbf{4}$ activates PP2A similarly to FTY720 (Fig. 3 ). We also performed a docking study (PDB code $2 \mathrm{E} 50)^{16)}$ to determine whether the aromatic head group of the derivative effectively binds to PP2A (Fig. 4). Similar to the interaction of a hydroxy group of FTY720 with Glu206 and Lys209, the phenol group of compound 4 showed the same interaction. In addition, the amide $\mathrm{C}=\mathrm{O}$ group in compound $\mathbf{4}$ interacts with Lys209. The aliphatic terminal chain of compound $\mathbf{4}$ interacts with Phe68, as like FTY720. However, none of the functional groups of compound 4 exhibited binding with Asp210 bound to the amine of FTY720. These results suggest that reducing the binding affinity causes low anticancer activity. In conclusion, our results demonstrate the importance of the substituted hydroxy group position in the aromatic head group and the necessity of introducing functional groups to replace the amine group of FTY720 for the design of new derivatives. We intend to further investigate the synthesis of additional derivatives employing this result, the inhibitory effect derivatives on sphingosine kinase, and the various biological activities such as the antituberculosis effect.

\section{Experimental}

General Experimental Procedures All chemicals were reagent grade and used as purchased. Flash column chromatography was performed on silica gel grade 60 (230-400 mesh). ${ }^{1} \mathrm{H}$ - and ${ }^{13} \mathrm{C}-\mathrm{NMR}$ spectra were recorded on a Bruker Avance I spectrometer, and chemical shifts are reported in $\delta$ 


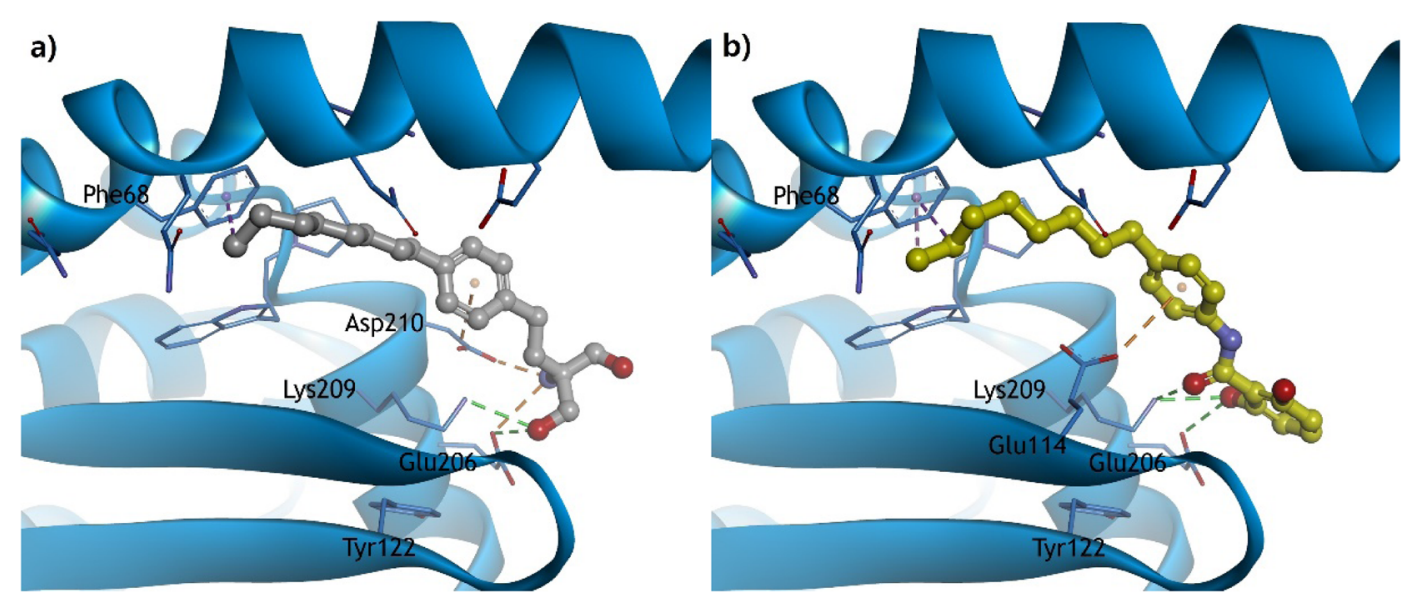

Fig. 4. Docking Model of (a) FTY720 (1, Gray, Ball and Stick) (b) Compound 4 (Yellow, Ball and Stick) in the I2PP2A/SET

The X-ray structure of IPP2A/SET was obtained from the Protein Data Bank (PDB code 2E50). The I2PP2A/SET is represented by blue ribbon model. The hydrogen bonds are shown as a green dashed line and electrostatic interactions are displayed as an orange dashed line. Also the hydrophobic interactions are shown as a pink dashed line. (Color figure can be accessed in the online version.)

units relative to deuterated solvents, which served as internal references, at 400 and $100 \mathrm{MHz}$, respectively. High resolution (HR) MS were recorded on an Agilent Technologies G6520A Q-TOF mass spectrometer using electrospray ionization (ESI).

General Procedure for Synthesis of FTY720 Derivatives 2-10 EDCI (3 eq), N,N-dimethyl-4-aminopyridine (DMAP) ( $0.5 \mathrm{eq})$, and the acid intermediates (1.5 eq) were added to a stirred solution of $4-n$-octylaniline $(100 \mathrm{mg}, 0.49 \mathrm{mmol})$ in $\mathrm{CH}_{2} \mathrm{Cl}_{2}(5 \mathrm{~mL})$. The reaction mixture was stirred under nitrogen for $12 \mathrm{~h}$. The solvent was removed in vacuo and the resulting residue was purified by flash column chromatography to afford the desired products $\mathbf{2}-\mathbf{1 0}$.

4-Hydroxy- $N$-(4-octylphenyl)benzamide 2

${ }^{1} \mathrm{H}-\mathrm{NMR} \quad\left(400 \mathrm{MHz}, \quad \mathrm{CDCl}_{3}-\mathrm{CD}_{3} \mathrm{OD}=3: 1 \quad(\mathrm{v} / \mathrm{v})\right) \quad \delta: 7.64$ (d, $J=8.0 \mathrm{~Hz}, 2 \mathrm{H}), 7.39(\mathrm{~d}, J=8.4 \mathrm{~Hz}, 2 \mathrm{H}), 7.04$ (t, $J=8.6 \mathrm{~Hz}$, $2 \mathrm{H}), 6.75(\mathrm{~d}, J=8.0 \mathrm{~Hz}, 2 \mathrm{H}), 2.44(\mathrm{t}, J=8.0 \mathrm{~Hz}, 2 \mathrm{H}), 1.44$ (q, $J=7.2 \mathrm{~Hz}, 2 \mathrm{H}), 1.25-1.02(\mathrm{~m}, 10 \mathrm{H}), 0.72(\mathrm{t}, J=6.0 \mathrm{~Hz}, 3 \mathrm{H})$; ${ }^{13} \mathrm{C}-\mathrm{NMR} \quad\left(100 \mathrm{MHz}, \mathrm{CDCl}_{3}-\mathrm{CD}_{3} \mathrm{OD}=3: 1 \quad(\mathrm{v} / \mathrm{v})\right) \quad \delta: \quad 167.1$, $160.7,139.4,136.0,129.5,129.0,126.1,121.0,115.5,35.6,32.1$, $31.8,29.9,29.7,29.5,29.5,22.9,14.2$; ESI-HR-MS $(\mathrm{M}+\mathrm{H})^{+}$ $\mathrm{m} / \mathrm{z}$ Calcd for $\mathrm{C}_{21} \mathrm{H}_{28} \mathrm{NO}_{2}$ 326.2120. Found 326.2144.

2,5-Dihydroxy- $N$-(4-octylphenyl)benzamide 3

Yellow oil; ${ }^{1} \mathrm{H}-\mathrm{NMR}\left(400 \mathrm{MHz}, \mathrm{CDCl}_{3}-\mathrm{CD}_{3} \mathrm{OD}=3: 1(\mathrm{v} / \mathrm{v})\right)$ $\delta$ : $7.38(\mathrm{~d}, J=8.4 \mathrm{~Hz}, 2 \mathrm{H}), 7.17(\mathrm{~d}, J=3.0 \mathrm{~Hz}, 1 \mathrm{H}), 7.05(\mathrm{~d}$, $J=8.6 \mathrm{~Hz}, 2 \mathrm{H}), 6.80(\mathrm{dd}, J=8.8,3.0 \mathrm{~Hz}, 1 \mathrm{H}), 6.70(\mathrm{~d}, J=8.8 \mathrm{~Hz}$, $1 \mathrm{H}), 2.46$ (t, $J=7.6 \mathrm{~Hz}, 2 \mathrm{H}), 1.47$ (q, $J=7.2 \mathrm{~Hz}, 2 \mathrm{H}), 1.28-1.07$ $(\mathrm{m}, 10 \mathrm{H}), 0.75(\mathrm{t}, J=6.9 \mathrm{~Hz}, 3 \mathrm{H}) ;{ }^{13} \mathrm{C}-\mathrm{NMR}\left(100 \mathrm{MHz}, \mathrm{CDCl}_{3}-\right.$ $\left.\mathrm{CD}_{3} \mathrm{OD}=3: 1(\mathrm{v} / \mathrm{v})\right) \delta: 167.1,152.3,149.2,139.7,135.0,128.8$, $121.7,121.5,118.2,116.6,113.4,35.4,31.8,31.5,29.4,29.2$, 22.6, 13.9; ESI-HR-MS $(\mathrm{M}+\mathrm{H})^{+} m / z$ Calcd for $\mathrm{C}_{21} \mathrm{H}_{28} \mathrm{NO}_{2}$ 326.2120. Found 326.2131.

2,6-Dihydroxy- $N$-(4-octylphenyl)benzamide 4

Yellow oil; ${ }^{1} \mathrm{H}-\mathrm{NMR} \quad\left(400 \mathrm{MHz}, \quad \mathrm{CDCl}_{3}-\mathrm{CD}_{3} \mathrm{OD}=3: 1\right.$ (v/v)) $\delta: 10.24(\mathrm{~s}, 3 \mathrm{H}, \mathrm{NH}), 7.49(\mathrm{~d}, J=8.3 \mathrm{~Hz}, 2 \mathrm{H}), 7.30(\mathrm{~d}$, $J=0.4 \mathrm{~Hz}, 1 \mathrm{H}), 7.21(\mathrm{dd}, J=8.3,1.9 \mathrm{~Hz}, 2 \mathrm{H}), 6.51(\mathrm{~d}, J=8.2 \mathrm{~Hz}$, $2 \mathrm{H}), 2.61(\mathrm{t}, J=7.8 \mathrm{~Hz}, 2 \mathrm{H}), 1.68-1.57(\mathrm{~m}, 2 \mathrm{H}), 1.41-1.23(\mathrm{~m}$, $10 \mathrm{H}), 0.92(\mathrm{t}, J=6.8 \mathrm{~Hz}, 3 \mathrm{H}) ;{ }^{13} \mathrm{C}-\mathrm{NMR}\left(100 \mathrm{MHz}, \mathrm{CDCl}_{3}-\right.$ $\left.\mathrm{CD}_{3} \mathrm{OD}=3: 1(\mathrm{v} / \mathrm{v})\right) \delta: 168.7,159.6,140.3,134.6,133.8,129.1$, 122.0, 108.9, 103.6, 35.6, 32.1, 31.7, 29.6, 29.4, 22.8, 14.3; ESIHR-MS $(\mathrm{M}+\mathrm{H})^{+} \mathrm{m} / z$ Calcd for $\mathrm{C}_{21} \mathrm{H}_{28} \mathrm{NO}_{2}$ 326.2120. Found 326.2182 .
2,4-Dihydroxy- $N$-(4-octylphenyl)benzamide 5

Yellow oil; ${ }^{1} \mathrm{H}-\mathrm{NMR}\left(400 \mathrm{MHz}, \mathrm{CDCl}_{3}-\mathrm{CD}_{3} \mathrm{OD}=3: 1\right.$ (v/v)) $\delta$ : $7.45(\mathrm{~d}, J=8.3 \mathrm{~Hz}, 2 \mathrm{H}), 7.14(\mathrm{~d}, J=8.2 \mathrm{~Hz}, 2 \mathrm{H}), 6.73$ (dd, $J=8.7,2.3 \mathrm{~Hz}, 1 \mathrm{H}), 6.41(\mathrm{dd}, J=8.8,2.4 \mathrm{~Hz}, 1 \mathrm{H}), 6.37(\mathrm{~d}$, $J=2.3 \mathrm{~Hz}, 1 \mathrm{H}), 2.54(\mathrm{t}, J=8.0 \mathrm{~Hz}, 2 \mathrm{H}), 1.59-1.49(\mathrm{~m}, 2 \mathrm{H})$, $1.23(\mathrm{~d}, J=13.8 \mathrm{~Hz}, 10 \mathrm{H}), 0.82(\mathrm{t}, J=6.4 \mathrm{~Hz}, 3 \mathrm{H}) ;{ }^{13} \mathrm{C}-\mathrm{NMR}$ $\left(100 \mathrm{MHz}, \mathrm{CDCl}_{3}-\mathrm{CD}_{3} \mathrm{OD}=3: 1(\mathrm{v} / \mathrm{v})\right) \delta: 168.3,165.0,154.5$, 140.2 , 134.6, 132.4, 129.0, 121.6, 113.0, 111.4, 103.0, 35.5, 32.0, 31.6, 29.6, 29.3, 22.7, 14.2; ESI-HR-MS (M+H) ${ }^{+} \mathrm{m} / z$ Calcd for $\mathrm{C}_{21} \mathrm{H}_{28} \mathrm{NO}_{2}$ 326.2120. Found 326.2128.

$\mathrm{N}$-(4-Octylphenyl)isonicotinamide 6

Brown oil; ${ }^{1} \mathrm{H}-\mathrm{NMR}\left(400 \mathrm{MHz}, \mathrm{CDCl}_{3}-\mathrm{CD}_{3} \mathrm{OD}=3: 1(\mathrm{v} / \mathrm{v})\right)$ $\delta: 8.49(\mathrm{~d}, J=5.8 \mathrm{~Hz}, 2 \mathrm{H}), 7.68(\mathrm{~d}, J=6.1 \mathrm{~Hz}, 2 \mathrm{H}), 7.37$ (d, $J=8.4 \mathrm{~Hz}, 2 \mathrm{H}), 6.96(\mathrm{~d}, J=8.4 \mathrm{~Hz}, 2 \mathrm{H}), 2.37(\mathrm{t}, J=7.6 \mathrm{~Hz}, 2 \mathrm{H})$, $1.45-1.32(\mathrm{~m}, 2 \mathrm{H}), 1.17-0.96(\mathrm{~m}, 10 \mathrm{H}), 0.64(\mathrm{t}, J=6.8 \mathrm{~Hz}, 3 \mathrm{H})$; ${ }^{13} \mathrm{C}-\mathrm{NMR} \quad\left(100 \mathrm{MHz}, \mathrm{CDCl}_{3}-\mathrm{CD}_{3} \mathrm{OD}=3: 1 \quad(\mathrm{v} / \mathrm{v})\right) \quad \delta: 160.5$, 139.1, 135.3, 135.2, 134.8, 128.7, 120.7, 120.0, 119.9, 35.2, 31.7, 31.4, 29.3, 29.1, 29.1, 22.5, 13.8; ESI-HR-MS $(\mathrm{M}+\mathrm{H})^{+} \mathrm{m} / \mathrm{z}$ Calcd for $\mathrm{C}_{20} \mathrm{H}_{27} \mathrm{~N}_{2} \mathrm{O}$ 311.2123. Found 311.2155.

4-Bromo- $N$-(4-octylphenyl)benzamide 7

Brown oil; ${ }^{1} \mathrm{H}-\mathrm{NMR}\left(400 \mathrm{MHz}, \mathrm{CDCl}_{3}-\mathrm{CD}_{3} \mathrm{OD}=3: 1(\mathrm{v} / \mathrm{v})\right)$ $\delta: 7.44(\mathrm{~d}, J=8.5 \mathrm{~Hz}, 2 \mathrm{H}), 7.25(\mathrm{~d}, J=8.4 \mathrm{~Hz}, 3 \mathrm{H}), 7.18(\mathrm{~d}$, $J=8.2 \mathrm{~Hz}, 2 \mathrm{H}), 6.80(\mathrm{~d}, J=8.2 \mathrm{~Hz}, 2 \mathrm{H}), 2.22(\mathrm{t}, J=7.6 \mathrm{~Hz}, 2 \mathrm{H})$, $1.28-1.18(\mathrm{~m}, 2 \mathrm{H}), 0.99-0.84(\mathrm{~m}, 10 \mathrm{H}), 0.50(\mathrm{t}, J=6.4 \mathrm{~Hz}$, $3 \mathrm{H}) ;{ }^{13} \mathrm{C}-\mathrm{NMR}\left(100 \mathrm{MHz}, \mathrm{CDCl}_{3}-\mathrm{CD}_{3} \mathrm{OD}=3: 1(\mathrm{v} / \mathrm{v})\right) \delta: 139.1$, $135.3,133.5,131.2,128.8,128.2,125.8,120.8,34.9,31.4,31.1$, 29.0, 28.8, 28.7, 22.1, 13.2; ESI-HR-MS (M+H) ${ }^{+} \mathrm{m} / \mathrm{z}$ Calcd for $\mathrm{C}_{21} \mathrm{H}_{27}$ BrNO 388.1276. Found 388.1219.

$\mathrm{N}$-(4-Octylphenyl)-1 $\mathrm{H}$-imidazole-5-carboxamide 8

Yellow oil; ${ }^{1} \mathrm{H}-\mathrm{NMR} \quad\left(400 \mathrm{MHz}, \quad \mathrm{CDCl}_{3}-\mathrm{CD}_{3} \mathrm{OD}=3: 1\right.$ $(\mathrm{v} / \mathrm{v})) \delta: 7.61(\mathrm{~s}, 1 \mathrm{H}), 7.59(\mathrm{~s}, 1 \mathrm{H}), 7.41(\mathrm{~d}, J=8.4 \mathrm{~Hz}, 2 \mathrm{H})$, $6.99(\mathrm{~d}, J=8.4 \mathrm{~Hz}, 2 \mathrm{H}), 2.41(\mathrm{t}, J=7.6 \mathrm{~Hz}, 2 \mathrm{H}), 1.48-1.36(\mathrm{~m}$, $2 \mathrm{H}), 1.21-1.02(\mathrm{~m}, 10 \mathrm{H}), 0.70(\mathrm{t}, J=6.9 \mathrm{~Hz}, 3 \mathrm{H}) ;{ }^{13} \mathrm{C}-\mathrm{NMR}$ $\left(100 \mathrm{MHz}, \mathrm{CDCl}_{3}-\mathrm{CD}_{3} \mathrm{OD}=3: 1(\mathrm{v} / \mathrm{v})\right) \delta: 160.5,139.1,135.3$, $135.2,134.8,128.7,120.7,120.0,119.9,35.2,31.7,31.4,29.3$, 29.1, 29.1, 22.5, 13.8; ESI-HR-MS $(\mathrm{M}+\mathrm{H})^{+} \mathrm{m} / \mathrm{z}$ Calcd for $\mathrm{C}_{18} \mathrm{H}_{26} \mathrm{~N}_{3} \mathrm{O}$ 300.2076. Found 300.2037.

2-(4-Hydroxyphenyl)- $N$-(4-octylphenyl)acetamide 9

Yellow oil; ${ }^{1} \mathrm{H}-\mathrm{NMR} \quad\left(400 \mathrm{MHz}, \quad \mathrm{CDCl}_{3}-\mathrm{CD}_{3} \mathrm{OD}=3: 1\right.$ $(\mathrm{v} / \mathrm{v})) \delta: 7.50(\mathrm{~d}, J=7.6 \mathrm{~Hz}, 2 \mathrm{H}), 7.27(\mathrm{~d}, J=8.3 \mathrm{~Hz}, 2 \mathrm{H}), 7.20$ (d, $J=8.3 \mathrm{~Hz}, 2 \mathrm{H}), 6.92(\mathrm{~d}, J=8.3 \mathrm{~Hz}, 2 \mathrm{H}), 2.65$ (t, $J=7.7 \mathrm{~Hz}$, 
$2 \mathrm{H}), 1.74-1.62(\mathrm{~m}, 2 \mathrm{H}), 1.47-1.32(\mathrm{~m}, 10 \mathrm{H}), 0.99(\mathrm{t}, J=6.6 \mathrm{~Hz}$, $3 \mathrm{H}) ;{ }^{13} \mathrm{C}-\mathrm{NMR}\left(100 \mathrm{MHz}, \mathrm{CDCl}_{3}-\mathrm{CD}_{3} \mathrm{OD}=3: 1\right.$ (v/v)) $\delta: 171.1$, $155.8,139.0,135.3,130.1,128.5,125.7,120.2,120.1,115.4$ $115.2,42.9,35.1,31.6,31.3,29.2,29.0,29.0,22.4$, 13.7; ESIHR-MS $(\mathrm{M}+\mathrm{H})^{+} \mathrm{m} / \mathrm{z}$ Calcd for $\mathrm{C}_{22} \mathrm{H}_{30} \mathrm{NO}_{2}$ 340.2277. Found 340.2282 .

(E)-3-(4-Chlorophenyl)- $N$-(4-octylphenyl)acrylamide $\mathbf{1 0}$

Slightly yellow oil; ${ }^{1} \mathrm{H}-\mathrm{NMR}\left(400 \mathrm{MHz}, \mathrm{CDCl}_{3}-\mathrm{CD}_{3} \mathrm{OD}=3: 1\right.$ (v/v)) $\delta: 7.64(\mathrm{~d}, J=15.7 \mathrm{~Hz}, 1 \mathrm{H}), 7.60(\mathrm{~d}, J=8.4 \mathrm{~Hz}, 2 \mathrm{H}), 7.49$ (d, $J=8.5 \mathrm{~Hz}, 2 \mathrm{H}), 7.35$ (d, $J=8.5 \mathrm{~Hz}, 2 \mathrm{H}), 7.17$ (d, $J=8.4 \mathrm{~Hz}$, $2 \mathrm{H}), 6.75(\mathrm{~d}, J=15.6 \mathrm{~Hz}, 1 \mathrm{H}), 2.60(\mathrm{t}, J=7.8 \mathrm{~Hz}, 2 \mathrm{H}), 1.68-1.57$ (m, 2H), 1.40-1.25 (m, 10H), $0.92(\mathrm{t}, J=6.8 \mathrm{~Hz}, 3 \mathrm{H}) ;{ }^{13} \mathrm{C}-\mathrm{NMR}$ $\left(100 \mathrm{MHz}, \mathrm{CDCl}_{3}-\mathrm{CD}_{3} \mathrm{OD}=3: 1(\mathrm{v} / \mathrm{v})\right) \delta: 164.7,139.9,139.0$, $135.8,135.3,133.3,128.9,128.8,128.6,121.7,120.0,35.25$, 31.7, 31.4, 29.3, 29.1, 22.5, 13.8; ESI-HR-MS $(\mathrm{M}+\mathrm{H})^{+} \mathrm{m} / \mathrm{z}$ Calcd for $\mathrm{C}_{23} \mathrm{H}_{29} \mathrm{ClNO}$ 370.1938. Found 370.1914.

Cell Culture and Proliferation Assays HT29 and AGS cells were maintained in Dulbecco's modified Eagle's medium (DMEM) supplemented with 10\% fetal bovine serum (FBS), $100 \mathrm{U} / \mathrm{mL}$ penicillin and $100 \mu \mathrm{g} / \mathrm{mL}$ streptomycin at $37^{\circ} \mathrm{C}$ in a humidified $5 \% \mathrm{CO}_{2}-95 \%$ air atmosphere. Cells were seeded in 96-well plates at a density of $3 \times 10^{3}$ cells $/ 100 \mu \mathrm{L} /$ well and incubated for $24 \mathrm{~h}$. The cells were then incubated in culture medium containing synthetic compounds $(20 \mu \mathrm{M})$. Following $24 \mathrm{~h}$ of incubation, the cell viability was determined using a EZ-CYTOX kit according to the manufacturer's protocol.

Annexin-V Staining Apoptosis was measured using an annexin-V fluorescein isothiocyanate (FITC) apoptosis detection kit, according to the manufacturer's instructions. Briefly, the cells were resuspended in binding buffer and then incubated with annexin-V-FITC and propidium iodide at room temperature. After incubation, stained cells were analyzed by Arthur $^{\mathrm{TM}}$ Image Based Cell Analyzer.

Molecular Modeling Molecular modeling studies of FTY720 and CPD 4 against the human I2PP2A/SET were performed using Schrödinger Suite 2017-2 (Schrödinger, LLC, http://www.schrodinger.com). The X-ray crystal structure obtained from the Protein Data Bank (http://www.rcsb.org/pdb) that PDB code is 2E50. The protein preparation was revised using Protein Preparation Wizard in Maestro v.11.2 and the receptor grid box was generated $25 \times 25 \times 25 \AA$ cubic size centered on complexed ligand. The ligands were minimized using OPLS 2005 force field with a dielectric constant value 80.0 in MacreoModel v.11.6. Flexible dockings were performed using the Glide v.7.5 program with standard precision method. The docking models of ligands were visualized using Discovery Studio 2016 (http://www.biovia.com).

PP2A Assay Activity was measured using a PP2A immunoprecipitation phosphatase assay kit (EMD Millipore Corporation) according to the manufacturer's instructions. Briefly, cell lysate was incubated with anti-PP2A and Protein
A agarose at $4^{\circ} \mathrm{C}$ and then had phosphopeptide added. After incubation, samples were analyzed by Thermo Scientific Multiskan GO.

Acknowledgments This research was supported by Basic Science Research Program through the National Research Foundation of Korea (NRF) funded by the Ministry of Science, ICT \& Future Planning (2015R1C1A1A01053302).

Conflict of Interest The authors declare no conflict of interest.

\section{References}

1) Azuma H., Takahara S., Ichimaru N., Wang J. D., Itoh Y., Otsuki Y., Morimoto J., Fukui R., Hoshiga M., Ishihara T., Nonomura N., Suzuki S., Okuyama A., Katsuoka Y., Cancer Res., 62, 1410-1419 (2002).

2) Matsuoka Y., Nagahara Y., Ikekita M., Shinomiya T., Br. J. Pharmacol., 138, 1303-1312 (2003).

3) Permpongkosol S., Wang J. D., Takahara S., Matsumiya K., Nonomura N., Nishimura K., Tsujimura A., Kongkanand A., Okuyama A., Int. J. Cancer, 98, 167-172 (2002).

4) Perrotti D., Neviani P., Lancet Oncol., 14, e229-e238 (2013).

5) Zhao Z., Choi J., Zhao C., Ma Z. A., J. Biol. Chem., 287, 5562-5573 (2012).

6) Moon H., Chon J., Joo J., Kim D., In J., Lee H., Park J., Choi J., Diabetes Metab. Res. Rev., 29, 19-24 (2013).

7) Turgut G. Ç., Doyduk D., Yıldırır Y., Yavuz S., Akdemir A., Dişli A., Şen A., Bioorg. Med. Chem., 25, 483-495 (2017).

8) Lim K. G., Sun C., Bittman R., Pyne N. J., Pyne S., Cell. Signal., 23, 1590-1595 (2011)

9) Pitman M., Costabile R. M., Pitson S. M., Cell. Signal., 28, 13491363 (2016)

10) Oh Y. S., Lee T., Shin S. M., Shrestha J., Lee D., Park E.-Y., Baek D. J., Bull. Korean Chem. Soc., 39, 261-264 (2018).

11) McCracken A. N., McMonigle R. J., Tessier J., Fransson R., Perryman M. S., Chen B., Keebaugh A., Selwan E., Barr S. A., Kim S. M., Roy S. G., Liu G., Fallegger D., Sernissi L., Brandt C., Moitessier N., Snider A. J., Clare S., Müschen M., Huwiler A., Kleinman M. T., Hanessian S., Edinger A. L., Leukemia, 31, 669-677 (2017).

12) Fransson R., McCracken A. N., Chen B., McMonigle R. J., Edinger A. L., Hanessian S., ACS Med. Chem. Lett., 4, 969-973 (2013).

13) Baek D. J., MacRitchie N., Anthony N. G., Mackay S. P., Pyne S., Pyne N. J., Bittman R., J. Med. Chem., 56, 9310-9327 (2013).

14) Imeri F., Fallegger D., Zivkovic A., Schwalm S., Enzmann G., Blankenbach K., Meyer zu Heringdorf D., Homann T., Kleuser B., Pfeilschifter J., Engelhardt B., Stark H., Huwiler A., Neuropharmacology, 85, 314-327 (2014).

15) Waisser K., Matyk J., Divišová H., Husáková P., Kuneš J. Klimešová V., Kaustová J., Möllmann U., Dahse H.-M., Miko M., Arch. Pharm. Chem. Life Sci., 339, 616-620 (2006).

16) Muto S., Senda M., Akai Y., Sato L., Suzuki T., Nagai R., Senda T., Horikoshi M., Proc. Natl. Acad. Sci. U.S.A., 104, 4285-4290 (2007). 